\title{
OPTICAL DETECTION OF RADIOCARBON DIOXIDE: FIRST RESULTS AND AMS INTERCOMPARISON
}

\author{
$\mathrm{I} \mathrm{Galli}^{1} \bullet \mathrm{S} \mathrm{Bartalini}^{1} \bullet \mathrm{P} \mathrm{Cancio}^{1} \bullet \mathrm{P}$ De Natale $^{1} \bullet \mathrm{D} \mathrm{Mazzotti}^{1,2} \bullet \mathrm{G} \mathrm{Giusfredi}^{1} \bullet \mathrm{M} \mathrm{E} \mathrm{Fedi}^{3} \bullet$ \\ P A Mandò 3
}

\begin{abstract}
The first results of an optical method for measuring radiocarbon concentrations, based on mid-infrared laser spectroscopy of a carbon dioxide gas sample, are presented with the theoretical bases explained in detail. The first measurements on modern and highly enriched samples show the extreme linearity of this technique over more than 5 decades. An intercomparison with accelerator mass spectrometry (AMS) is performed both for modern and ${ }^{14} \mathrm{C}$-dead samples, assessing the almost perfect agreement of their respectively measured concentration values. The main features of our technique are compared with liquid scintillation counting (LSC) and AMS, and future developments of the current setup are discussed.
\end{abstract}

\section{INTRODUCTION}

Radiocarbon $\left({ }^{14} \mathrm{C}\right.$ ) is a peculiar isotope, its isotopic concentration being about 1 part per trillion (ppt, $10^{-12}$ ). Notwithstanding this low concentration, for more than $60 \mathrm{yr}{ }^{14} \mathrm{C}$ has been the "natural clock" for dating samples that contain organic matter. For the first $30 \mathrm{yr}$, liquid scintillation counting (LSC), requiring comparably large carbon mass amounts and long measurement times, had been the only method for dating samples. For the last $30 \mathrm{yr}$, accelerator mass spectrometry (AMS; Bennett et al. 1977; Synal and Wacker 2010) has been adopted as the standard method, requiring much smaller carbon mass amounts and much shorter measurement times, but also large, expensive, and highmaintenance facilities (although, in the last few years, a big effort has been made towards lowenergy and smaller machines).

Just a few years after the onset of the AMS-based dating method, scientists from a world quite different from nuclear physics began to study the feasibility of ${ }^{14} \mathrm{C}$ dating by infrared (IR) laser absorption spectroscopy (Labrie and Reid 1981). Some years later, following a parallel road, precise measurements of the ${ }^{13} \mathrm{CO}_{2} /{ }^{12} \mathrm{CO}_{2}$ isotopic ratio (Murnick and Peer 1994) were demonstrated by an analytical apparatus called a laser-assisted ratio analyzer (LARA), based on the optogalvanic spectroscopic technique. A more recent improvement of that technique, intracavity optogalvanic spectroscopy (ICOGS) (Murnick et al. 2008, 2010), has achieved the extremely high sensitivity needed for detection of ${ }^{14} \mathrm{C}$-labeled carbon dioxide. Unfortunately, one of the main limitations of ICOGS is its non-linear calibration for concentrations $>1.5 \mathrm{ppt}$.

Following the initial work by Labrie and Reid (1981), we succeeded in measuring, for the first time, the spectral area (and hence the absolute radiocarbon dioxide $\left[{ }^{14} \mathrm{C}^{16} \mathrm{O}_{2}\right]$ concentration) of the $\left(00^{0} 1-00^{0} 0\right) \mathrm{P}(20)$ line of the ${ }^{14} \mathrm{C}^{16} \mathrm{O}_{2}$ isotopologue. This result was obtained by exploiting the unique features of a highly performing mid-IR coherent light source, combined with the strong molecular absorption of carbon dioxide in the mid-IR. A newly developed laser spectroscopy technique allowed us to achieve the extreme sensitivity necessary to detect the ${ }^{14} \mathrm{C}^{16} \mathrm{O}_{2}$ molecules at their tiny natural abundance with an all-optical setup that is much more compact and less expensive than AMS (Galli et al. 2011). The new spectroscopic technique, named SCAR (saturated-

\footnotetext{
${ }^{1}$ Istituto Nazionale di Ottica (INO) - CNR, European Laboratory for Non-linear Spectroscopy (LENS), Via Carrara 1, 50019 Sesto Fiorentino FI, Italy.

${ }^{2}$ Corresponding author: davide.mazzotti@ino.it.

${ }^{3}$ Istituto Nazionale di Fisica Nucleare (INFN) - Sez. Firenze, Dipartimento di Fisica e Astronomia, Via Sansone 1, 50019 Sesto Fiorentino FI, Italy.
}

(c) 2013 by the Arizona Board of Regents on behalf of the University of Arizona Proceedings of the 21st International Radiocarbon Conference edited by A J T Jull \& C Hatté RADIOCARBON, Vol 55, Nr 2-3, 2013, p 213-223 


\section{Galli et al.}

absorption cavity ring-down; Giusfredi et al. 2010) makes use of saturation of molecular absorption to enhance resolution (up to 3 orders of magnitude) and sensitivity ( $\sim 20$ times) with respect to conventional cavity ring-down spectroscopy (Romanini et al. 1997). Combining SCAR with a frequency-comb-linked difference-frequency-generated (DFG) coherent source, delivering up to $30 \mathrm{~mW}$ continuous-wave (CW) radiation tunable around 4.5- $\mu \mathrm{m}$ wavelength (Galli et al. 2010), allowed us to set an unprecedented limit in trace gas detection, accessing the part-per-quadrillion (ppq, $10^{-15}$ ) concentration range. SCAR-based results are still 1 order of magnitude below those of AMS, but this technique has ample room for improvement. Finally, as we will show in this work, the SCAR dynamic range encompasses more than 5 orders of magnitude in measurable concentration values. This range is much wider than in AMS setups, which are typically limited to 3 orders of magnitude, even when designed for pharmaceutical applications (Schulze-König et al. 2010).

\section{METHODS}

Our method for ${ }^{14} \mathrm{C}^{16} \mathrm{O}_{2}$ optical detection is based on molecular absorption spectroscopy and on a recently developed high-sensitivity technique for trace gas sensing, saturated-absorption cavity ring-down (SCAR, discussed above). The SCAR working principle can be shortly described as follows. An optical cavity filled with $\mathrm{CO}_{2}$ is illuminated with an intense $\mathrm{CW}$ laser tuned to excite the targeted $\left(00^{0} 1-00^{0} 0\right) \mathrm{P}(20)$ ro-vibrational transition of ${ }^{14} \mathrm{C}^{16} \mathrm{O}_{2}$. The specific resonance line was selected following dual criteria: maximum absorption coefficient and minimum level of interferences from all other nearby lines. When the laser is turned off, photons stored in the cavity decay due to both ${ }^{14} \mathrm{C}^{16} \mathrm{O}_{2}$ absorption and mirror leakage. Because light intensity saturates the absorption of the ${ }^{14} \mathrm{C}^{16} \mathrm{O}_{2}$ molecule, the initial decay characteristic is affected by losses from the mirrors only. Once this background is subtracted, we can determine the absolute quantity of ${ }^{14} \mathrm{C}^{16} \mathrm{O}_{2}$ from the linear molecular absorption encoded in the decay tail.

\section{Basic Molecular Absorption Spectroscopy}

Our method is based on the measurement of a particular molecular absorption spectrum: ${ }^{14} \mathrm{C}^{16} \mathrm{O}_{2}$ concentrations retrieved from recorded spectral areas. We now recall some basic concepts of molecular absorption spectroscopy, aiming at finally revealing the rigorous link existing between these 2 physical quantities. In the following, we will enclose in brackets the units of the physical quantities and we will use some non-SI units common in the molecular spectroscopy community and used e.g. in the molecular database HITRAN (Rothman et al. 2009).

When electromagnetic radiation interacts with a molecular gas, the strongest absorption of photons, as allowed by selection rules, is due to the electric dipole moment of the molecular transition. Let $l$ and $u$ represent the lower and upper state quantum numbers, respectively. The transition linestrength $S_{l u}[\mathrm{~cm}]$ from state $|l\rangle$ to state $|u\rangle$ can then be expressed as:

$$
S_{l u}(T) \propto|\langle u|\mu| l\rangle|^{2} g_{l} \frac{\left(e^{-\frac{E_{l}}{k T}}-e^{\frac{E_{u}}{k T}}\right)}{Z(T)} ; \quad Z(T) \equiv \sum_{n} g_{n} e^{-\frac{E_{n}}{k T}}
$$

where $T[\mathrm{~K}]$ is the absolute temperature, $\mu[\mathrm{D}]^{4}$ is the electric dipole moment, $Z$ is the thermodynamic partition function, and $g_{n}$ and $E_{n}[\mathrm{~J}]$ are the degeneracy and energy of state $|n\rangle$, respectively.

${ }^{4} \mathrm{D}=$ Debye $\left(\approx 3.336 \times 10^{-30} \mathrm{C} \cdot \mathrm{m}\right)$. 


\section{Optical Detection of Radiocarbon Dioxide}

We will assume to have a gas mixture with different components, and all physical quantities related to them will be labeled by an integer number $i$. Let $i=0$ label the only absorbing gas, whose electric dipole transition is resonant with the electromagnetic radiation interacting with it (the ${ }^{14} \mathrm{C}^{16} \mathrm{O}_{2}$ isotopologue, in our case), and $i \geq 1$ label all the non-absorbing gases in the mixture (all other $\mathrm{CO}_{2}$ isotopologues, in our case). The absorbing gas density $n_{0}\left[\mathrm{~cm}^{-3}\right]$ can be expressed in terms of thermodynamic quantities as:

$$
n_{0} \equiv \frac{N_{0}}{V}=n_{s} \frac{P_{0}}{P_{s}} \frac{T_{s}}{T}
$$

where $N_{0}$ is the absorbing gas number of molecules, $V$ is the volume $\left[\mathrm{cm}^{3}\right], P_{0}$ is the absorbing gas partial pressure [atm], and $P_{s} \equiv 1 \mathrm{~atm}$ and $T_{s} \equiv 296 \mathrm{~K}$ are standard pressure and temperature, respectively. The Loschmidt density $n_{s}$ is a constant defined as:

$$
n_{s} \equiv \frac{P_{s}}{k T_{s}} \approx 2.479 \times 10^{19} \mathrm{~cm}^{-3}
$$

and represents the density of an ideal gas at standard thermodynamic conditions.

The molecular absorption cross-section $\sigma_{l u}\left[\mathrm{~cm}^{2}\right]$ from state $|l\rangle$ to state $|u\rangle$ is defined as:

$$
\sigma_{l u}\left(v ; P_{i}, T\right) \equiv S_{l u}(T) g\left(v ; P_{i}, T\right) ; \quad \int g\left(v ; P_{i}, T\right) \mathrm{d} v=1
$$

where $v$ is the radiation frequency $\left[\mathrm{cm}^{-1}\right], P_{i}$ is the partial pressure of gas $i$ [atm], and $g$ is the lineshape function $[\mathrm{cm}]$, with normalization to 1 . The lineshape of a molecular absorption spectrum is related to the broadening mechanisms for the transition. In the low-pressure regime, when the Doppler broadening is dominant, the transition is inhomogeneous and $g$ has Gaussian lineshape, named $g_{D}$, which is defined as:

$$
g_{D}(v ; T) \equiv \frac{1}{\Gamma_{D}(T)} \sqrt{\frac{\ln 2}{\pi}} e^{-\ln 2\left[\frac{v-v_{l u}}{\Gamma_{D}(T)}\right]^{2}} ; \quad \Gamma_{D}(T)=v_{l u} \sqrt{2 \ln 2 \frac{k T}{m c^{2}}}
$$

where $\Gamma_{D}$ is the Doppler linewidth ${ }^{5}\left[\mathrm{~cm}^{-1}\right], v_{l u}$ is the transition frequency $\left[\mathrm{cm}^{-1}\right]$, and $m$ is the molecular mass $[\mathrm{kg}]$. In the high-pressure regime, when the collisional broadening is dominant, the transition is homogeneous and $g$ has a Lorentzian lineshape, named $g_{L}$, which is defined as:

$$
g_{L}\left(v ; P_{i}, T\right) \equiv \frac{1}{\pi \Gamma_{L}\left(P_{i}, T\right)} \frac{1}{1+\left[\frac{v-v_{l u}}{\Gamma_{L}\left(P_{i}, T\right)}\right]^{2}} ; \quad \Gamma_{L}\left(P_{i}, T\right)=\sum_{i=0} \gamma_{i}(T) P_{i}
$$

where $\Gamma_{L}$ is the Lorentzian linewidth $\left[\mathrm{cm}^{-1}\right]$ and $\gamma_{i}$ is the pressure broadening coefficient of the gas $i\left[\mathrm{~cm}^{-1} \cdot \mathrm{atm}^{-1}\right]$. In the intermediate regime, when Doppler and collisional broadenings are comparable, the transition can be approximated by a Voigt lineshape, named $g_{b}$, which is defined as:

\footnotetext{
${ }^{5}$ All linewidths are to be understood as half-width at half-maximum (HWHM) values.
} 


\section{Galli et al.}

$$
g_{V}\left(v ; P_{i}, T\right) \equiv \frac{1}{\Gamma_{L}\left(P_{i}, T\right) \Gamma_{D}(T)} \sqrt{\frac{\ln 2}{\pi^{3}}} \int \frac{e^{-\ln 2\left[\frac{v^{\prime}-v_{l u}}{\Gamma_{D}(T)}\right]^{2}}}{1+\left[\frac{v-v^{\prime}}{\Gamma_{L}\left(P_{i}, T\right)}\right]^{2}} \mathrm{~d} v^{\prime}
$$

which is simply a mathematical convolution of $g_{D}$ and $g_{L}\left(g_{V} \equiv g_{D} * g_{L}\right)$.

The physical quantity measured by any absorption spectroscopy technique is the linear absorption coefficient $\alpha\left[\mathrm{cm}^{-1}\right]$, which can be expressed as:

$$
\alpha\left(v ; P_{i}, T\right) \equiv n_{0} \sigma_{l u}\left(v ; P_{i}, T\right)=n_{s} \frac{P_{0}}{P_{s}} \frac{T_{s}}{T} S_{l u}(T) g\left(v ; P_{i}, T\right)
$$

Hence, the absorbing gas concentration $P_{0} / P$, which is the final goal of the measurement, can be retrieved from this equation:

$$
\frac{P_{0}}{P}=\frac{1}{n_{s}} \frac{P_{s}}{P} \frac{T}{T_{s}} \frac{1}{S_{l u}} \int \alpha\left(v ; P_{i}, T\right) \mathrm{d} v
$$

Just for convenience, in the following we will plot all absorption spectra in terms of the gas-induced decay rate $\gamma_{g} \equiv c \alpha$, which is the value really measured by the experiment, instead of the absorption coefficient $\alpha$.

\section{SCAR Setup}

The experimental apparatus we used for ${ }^{14} \mathrm{C}^{16} \mathrm{O}_{2}$ optical detection has already been fully described in Galli et al. (2010, 2011). Its simplified schematics are shown in Figure 1. Here, we will focus on describing in detail the measurement cell and the gas sample filling it. The measurement cell is a cylindrical vacuum chamber $1.2 \mathrm{~m}$ long and $10 \mathrm{~cm}$ in internal diameter. It is enclosed in a polystyrene box, which can be filled with dry-ice pellets for cooling the chamber down to $195 \mathrm{~K}$. The chamber houses a Fabry-Perot optical resonator resting inside of it on 4 cantilevered legs, which dampen vibrations (for frequencies $>20 \mathrm{~Hz}$ ) in all 3 spatial directions. The mechanical frame of the optical resonator is made of 3 Invar bars connected by 2 circular flanges, leaving $8 \mathrm{~L}$ internal volume available for the gas. At both ends of the frame, properly machined flanges house the mirrors, with highreflectivity dielectric coatings at $4.5 \mu \mathrm{m}$ wavelength. The total losses for each mirror (transmission plus absorption/scattering) amount to $\sim 270$ parts per million ( $\mathrm{ppm}$ ) and the achieved optical finesse is higher than 11,000. The mirror mounting flanges have screws for coarse alignment and PZT for fine adjustment of both the alignment and the cavity length. The mirror spacing is $1 \mathrm{~m}$ and the corresponding free spectral range is $150 \mathrm{MHz}$.

The gas sample is carbon dioxide at total pressure $P=12 \mathrm{mbar}$ and temperature $T=195 \mathrm{~K}$ (corresponding to $\sim 0.15 \mathrm{~L}$ volume at standard thermodynamic conditions). This pressure is a trade-off between 2 competing goals: maximizing the spectral area of the target line, which is proportional to the absorbing gas partial pressure (and thus to $P$ ); and minimizing interferences from tails of nearby lines of other $\mathrm{CO}_{2}$ isotopologues and/or different molecular components, which are roughly proportional to $P^{2}$. This trade-off value also determines the lineshape, which, in this case, is a Voigt profile (see Equation 7) with both Doppler and Lorentzian widths of about $50 \mathrm{MHz}$. The reason for cooling the gas sample down to $195 \mathrm{~K}$ is a strong decrease in the linestrength of the nearby $\left(05^{5} 1-05^{5} 0\right)$ $\mathrm{P}(19)$ e line of ${ }^{13} \mathrm{C}^{16} \mathrm{O}_{2}$ (more than 3 orders of magnitude). This line is blue-shifted by $\sim 230 \mathrm{MHz}$ with respect to the target one, so its red tail partially overlaps with the blue tail of the $\mathrm{P}(20)$ and must 


\section{Optical Detection of Radiocarbon Dioxide}

be taken into account to avoid systematic overestimation of the $\mathrm{P}(20)$ spectral area. Another line of a different kind lies almost at the same frequency of the $\mathrm{P}(19) \mathrm{e}$, namely the $\left(01^{1} 1-01^{1} 0\right) \mathrm{Q}(12) \mathrm{e}$ line of ${ }^{14} \mathrm{~N}_{2}{ }^{16} \mathrm{O}$. Its linestrength is so high (and depending not very much on temperature) that a $\mathrm{N}_{2} \mathrm{O}$ concentration below $0.3 \mathrm{ppb}$ is required to produce a negligible interference with the target line.

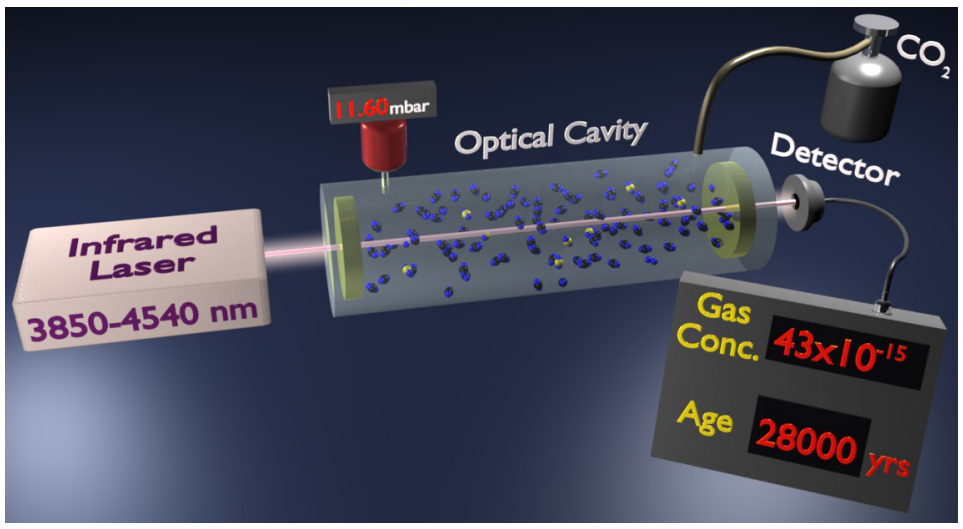

Figure 1 Experimental setup

\section{AMS Setup}

AMS measurements were performed at the INFN-LABEC laboratory in Florence, using the AMSdedicated beam line installed at the 3MV Tandetron accelerator (Fedi et al. 2007). In brief, the AMS beam line is equipped with a multisample Cs sputtering ion source, an electrostatic analyzer, and a bouncer injector on the low-energy side. At the accelerator terminal, typically kept at $2.5 \mathrm{MV}$ during ${ }^{14} \mathrm{C}$ measurements, stripping is achieved thanks to interaction with argon. On the high-energy side, charge $3+$ is analyzed through a system consisting in a magnet and an additional electrostatic analyzer. To count the rare isotope, either a simple silicon PIN photodiode (Hamamatsu S3590-09) or a recently installed time-of-flight (TOF) system can be used. For these measurements, ${ }^{14} \mathrm{C}$ ions were counted by the photodiode.

\section{RESULTS}

The ${ }^{14} \mathrm{C}^{16} \mathrm{O}_{2}$ concentration measurement was performed for 2 particular $\mathrm{CO}_{2}$ gas samples: one from a high-purity industrial gas cylinder, and another one produced by fermentation of brown cane sugar (purchased in year 2010) dissolved in water (in a bottle with no air content) with yeast. The former is equivalent to a ${ }^{14} \mathrm{C}$-dead sample, being the gas of fossil origin, while the latter one is obviously a modern ${ }^{14} \mathrm{C}$ sample.

\section{SCAR Measurements}

We have measured the absorption spectrum of the ${ }^{14} \mathrm{C}^{16} \mathrm{O}_{2}$ target line with the same procedure already described in Galli et al. (2011). Its frequency span has been extended from 400 up to $740 \mathrm{MHz}$, by performing 2 partially overlapped frequency scans across the line, one red-shifted and the other one blue-shifted with respect to the line center. The recorded spectrum is shown in Figure 2. If we compare it with Figure 2 of Galli et al. (2011), we can clearly see that the interfering line from the ${ }^{13} \mathrm{C}^{16} \mathrm{O}_{2}$ isotopologue was not visible in the previous measurement, due to the earlier only red-shifted narrower scan. Indeed, its spectral area is comparable to the target line. Therefore, working at the current gas temperature of $195 \mathrm{~K}$ requires a careful fitting of both the target line and 


\section{Galli et al.}

the interfering one, limiting the achievable precision and accuracy for the retrieved spectral area of the former one (and hence of the ${ }^{14} \mathrm{C}$ concentration). To eliminate this limitation, we decided to further lower the working temperature in a future version of the SCAR setup, as we will discuss below.

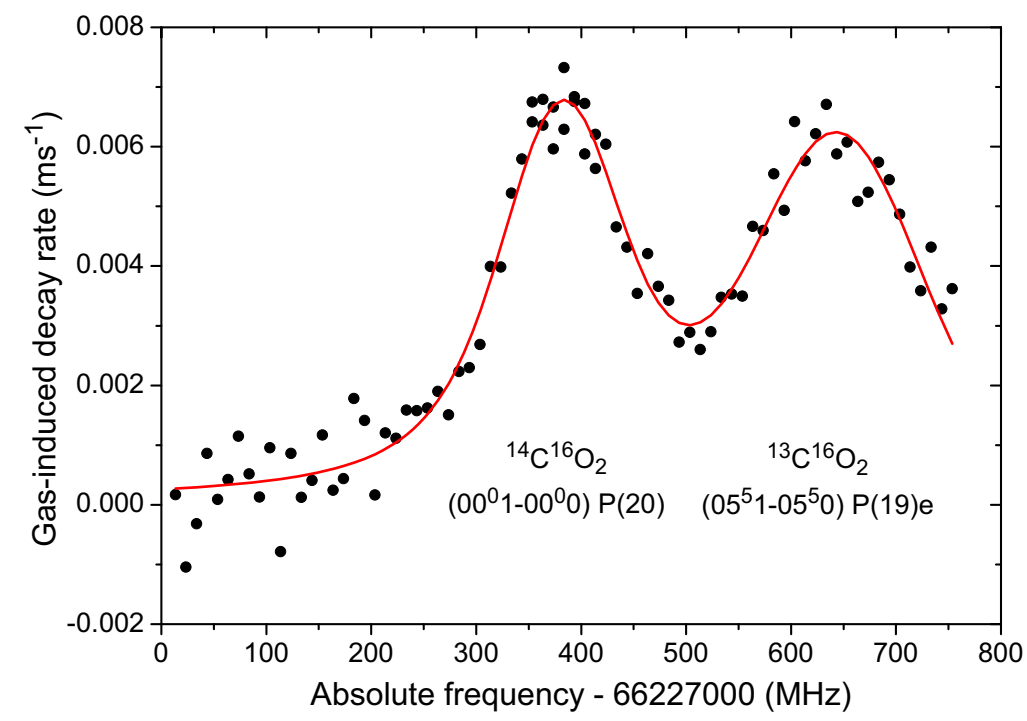

Figure 2 Absorption spectrum of a modern $\mathrm{CO}_{2}$ sample recorded at $195 \mathrm{~K}$ with the SCAR technique around the $\left(00^{0} 1-00^{0} 0\right) \mathrm{P}(20)$ line of ${ }^{14} \mathrm{C}^{16} \mathrm{O}_{2}$. The $\left(05^{5} 1-05^{5} 0\right) \mathrm{P}(19)$ e line of ${ }^{13} \mathrm{C}^{16} \mathrm{O}_{2}$ is clearly visible at the blue side. The sum of 2 Voigt profiles (red line) fits experimental data (black points).

Another issue is the linearity of the measured spectral area vs. ${ }^{14} \mathrm{C}$ concentration, which was already demonstrated in Galli et al. (2011) in the range below natural abundance. We have performed a run of measurements to test such linearity with highly enriched samples. Results are plotted in Figure 3.

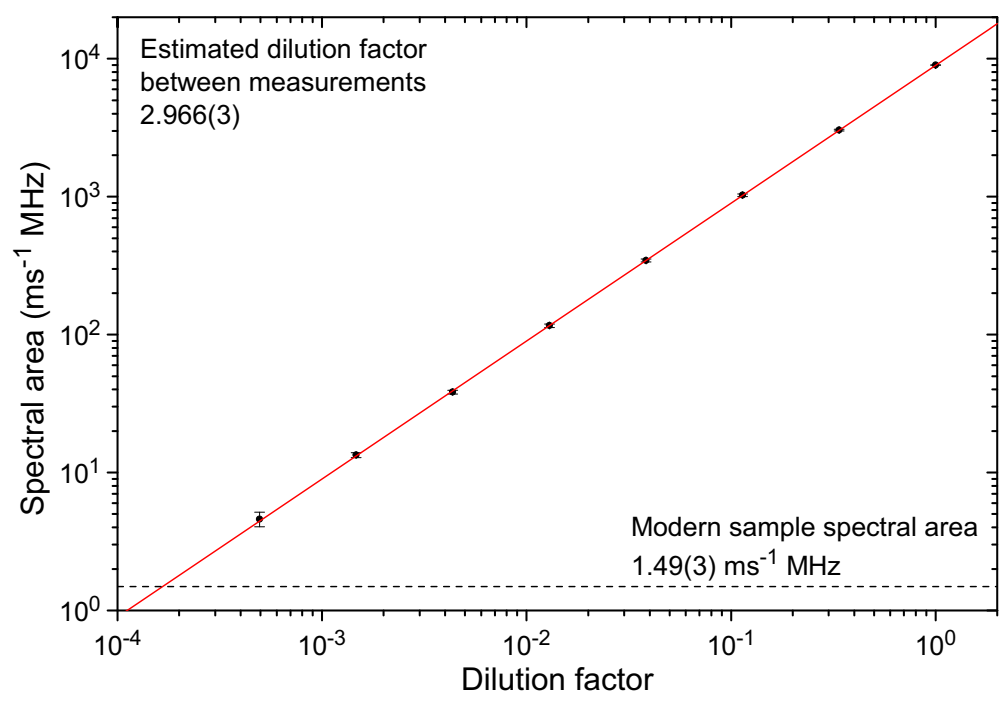

Figure 3 Detection linearity for enriched samples, over more than 3 orders of magnitude 


\section{Optical Detection of Radiocarbon Dioxide}

We started with a $\mathrm{CO}_{2}$ sample with ${ }^{14} \mathrm{C}$ enrichment of $\sim 6400$ modern fraction. This initial sample was obtained by diluting in argon an almost pure ${ }^{14} \mathrm{CO}_{2}$ commercial sample (American Radiolabeled Chemicals Inc, prod. ARC 0307) contained in a 9-mL glass vial. The resulting concentration of the ${ }^{14} \mathrm{CO}_{2}$ molecules contained in the mixture was about $1 \mathrm{ppm}$. We further diluted this mixture about 100 times with dead $\mathrm{CO}_{2}$ from the gas cylinder. We recorded 8 spectra, each time diluting the initial sample by a factor very close to 3 (the real value is shown in the linear fit, as a free parameter). As seen in Figure 3, the spectral area (and hence the measured ${ }^{14} \mathrm{C}$ concentration) is strictly proportional to the dilution factor (and hence to the real ${ }^{14} \mathrm{C}$ concentration).

\section{AMS Measurements}

As far as sample preparation is concerned (Fedi et al. 2013), cathodes from cane sugar were produced by combusting the raw material in a CN elemental analyzer (Thermo EA1200) and then converting the evolved $\mathrm{CO}_{2}$ to graphite by reaction with hydrogen, at high temperature $\left(600{ }^{\circ} \mathrm{C}\right)$ and in the presence of iron (Alfa Aesar iron, $10 \mu \mathrm{m}$ average size) as catalyst. Carbon dioxide collected from the industrial gas cylinder was directly transferred into the graphitization line and then converted to graphite as already discussed. The cathode mass was $\sim 0.7 \mathrm{mg}$ carbon mixed with $3 \mathrm{mg}$ of iron.

${ }^{14} \mathrm{C} /{ }^{12} \mathrm{C}$ measured isotopic ratios in the intercomparison samples were corrected for isotopic fractionation, considering the ${ }^{13} \mathrm{C} /{ }^{12} \mathrm{C}$ ratios also measured along the beam line (in fact ${ }^{12} \mathrm{C}$ and ${ }^{13} \mathrm{C}$ currents are measured in 2 off-line Faraday cups installed at the exit of the analyzing magnet) and for background counts. Finally, they were normalized to ${ }^{14} \mathrm{C} /{ }^{12} \mathrm{C}$ isotopic ratios measured in a set of samples prepared from the standard NIST oxalic acid II (NIST SRM 4990C).

\section{SCAR-AMS Intercomparison}

We performed an intercomparison between the SCAR and AMS techniques, by measuring both samples with both techniques, and these SCAR-AMS intercomparison results are summarized in Table $1 .{ }^{14} \mathrm{C}$ content is expressed in percent modern carbon (pMC) units for AMS measurements, while natural units for SCAR are $\mathrm{ms}^{-1} \mathrm{MHz}$, representing the spectral area of the ${ }^{14} \mathrm{C}^{16} \mathrm{O}_{2}$ targeted line (see Figure 2). We observe a perfect agreement, within their respective uncertainties, between the ${ }^{14} \mathrm{C}$ concentration values measured by the 2 techniques for both samples. This is highly encouraging, considering that only a theoretical estimation for the linestrength of the target line $\left(S=3.1 \times 10^{-18} \mathrm{~cm}\right.$ at $195 \mathrm{~K}$, see Ref. 23 in Galli et al. 2011) was used to retrieve the ${ }^{14} \mathrm{C}^{16} \mathrm{O}_{2}$ concentration from Equation 9. We would like to point out that, in any case, an accurate knowledge of the linestrength value will not be needed when comparing an unknown sample with a modern standard, like AMS and LSC do. Indeed, by applying the same Equation 9 both to the sample and to the standard and taking the ratio of the 2 retrieved concentrations (yielding the pMC value for the sample), the linestrength parameter will be canceled out.

Table 1 AMS-SCAR intercomparison.

\begin{tabular}{lrr|rr}
\hline & \multicolumn{2}{c|}{ AMS } & \multicolumn{2}{c}{ SCAR } \\
\cline { 2 - 5 } & \multicolumn{1}{c}{ pMC } & \multicolumn{1}{c}{$\mathrm{ppq}$} & \multicolumn{1}{c}{$\mathrm{ms}^{-1} \cdot \mathrm{MHz}$} & \multicolumn{1}{c}{$\mathrm{ppq}$} \\
\hline Modern $\mathrm{CO}_{2}$ & $106.68 \pm 0.37$ & $1253.3 \pm 7.8$ & $1.491 \pm 0.026$ & $1248 \pm 22$ \\
Fossil $\mathrm{CO}_{2}$ & $0.26 \pm 0.05$ & $3.1 \pm 0.6$ & $-0.004 \pm 0.036$ & $-4 \pm 30$ \\
\hline
\end{tabular}




\section{Galli et al.}

\section{DISCUSSION}

\section{Competing Techniques}

SCAR features are compared with the 2 main competing techniques, LSC and AMS, in Table 2. The major differences between SCAR and the other techniques lie in the detection method and the sample material. LSC counts electrons with few hundreds $\mathrm{keV}$ energy emitted in radioactive $\beta$ decays of ${ }^{14} \mathrm{C}$ nuclei, by detecting photon bursts generated by a liquid scintillator mixed with the benzene sample. AMS counts the carbon ions extracted from the accelerator source from the sample reduced to graphite (or, in a few facilities, to gaseous carbon dioxide); ions are accelerated up to few $\mathrm{MeV}$ energy and finally counted on a particle detector at the end of the accelerator beam line. SCAR detects mid-IR photons with $0.25 \mathrm{eV}$ energy absorbed by the low-pressure carbon dioxide sample filling an optical cavity.

Table 2 Comparison of competing techniques.

\begin{tabular}{|c|c|c|c|}
\hline & LSC & AMS & SCAR \\
\hline${ }^{14} \mathrm{C}$ detection method & $\beta$-decay count & ${ }^{14} \mathrm{C}$ ion count & Absorbed photons \\
\hline Sample material & $\mathrm{C}_{6} \mathrm{H}_{6}$ & $\mathrm{C}+\mathrm{Fe}$ & $\mathrm{CO}_{2}$ \\
\hline $\mathrm{C}$ mass $(\mathrm{mg})$ & $\sim 4000$ & $<1$ & $\sim 70$ \\
\hline Measurement time (hr) & $\sim 17$ & $\sim 1$ & $\sim 3$ \\
\hline Measurement repeatability & Same technique & Same technique & Any technique \\
\hline Interferences and/or background & Cosmic rays & $\begin{array}{l}{ }^{14} \mathrm{~N} \\
{ }^{13} \mathrm{CH} \\
{ }^{12} \mathrm{CH}_{2}\end{array}$ & $\begin{array}{l}{ }^{13} \mathrm{C}^{16} \mathrm{O}_{2}(\mathrm{~T}>170 \mathrm{~K}) \\
\mathrm{N}_{2} \mathrm{O}(>0.3 \mathrm{ppb}) \\
\mathrm{O}_{3}(>10 \mathrm{ppm})\end{array}$ \\
\hline Precision for modern (pMC) & $\sim 0.5$ & $\sim 0.3$ & $\sim 2$ \\
\hline Limiting factor & Poisson statistics & Poisson statistics & $\alpha_{\min }$ \\
\hline Footprint $\left(\mathrm{m}^{2}\right)$ & $\sim 1$ & $7-200$ & $\sim 2$ \\
\hline Cost (in thousands $€$ ) & $\sim 120$ & $300-3000$ & $\sim 300$ \\
\hline
\end{tabular}

Different carbon mass amounts and measurement times are required for the 3 techniques. LSC needs $\sim 4 \mathrm{~g}$ carbon and $\sim 17 \mathrm{hr}$, SCAR needs $\sim 70 \mathrm{mg}$ carbon and $\sim 3 \mathrm{hr}$, while AMS is the least demanding both in terms of carbon mass, $<1 \mathrm{mg}$, and time, $\sim 1 \mathrm{hr}$. With SCAR, the measurement on a given sample can be repeated with any of the 3 techniques, while with LSC and AMS this repeatability is restricted to the same technique only. This fact is due to the sample preparation, which is simpler and shorter for SCAR, requiring just the combustion and carbon dioxide extraction, while AMS needs a further graphitization (with the only exception of the gaseous-source-based facilities), and LSC needs more chemical-reaction steps to produce the benzene sample. Also, with SCAR the gas sample is neither altered nor destroyed by the measurement process, since it just relies on absorption (and re-emission) of mid-IR photons from ${ }^{14} \mathrm{C}^{16} \mathrm{O}_{2}$ molecules.

The 3 techniques have different interferences and/or background disturbing their measurement process. LSC suffers from spurious counts due to cosmic rays, which require massive lead shields to be reduced down to an acceptable level. When ${ }^{14} \mathrm{C}$ atoms are directly counted, as in an AMS measurement, the interfering atoms are the isobars ${ }^{14} \mathrm{~N},{ }^{13} \mathrm{CH}$, and ${ }^{12} \mathrm{CH}_{2}$ : the former is just suppressed in the accelerator source, thanks to its negative electron affinity; the latter two are reduced to negligible levels thanks to the stripping process at the accelerator terminal. The target spectroscopic line used by SCAR to detect the ${ }^{14} \mathrm{C}^{16} \mathrm{O}_{2}$ isotopologue has several interfering lines from different molecules, all lying at the blue side of the $\mathrm{P}(20)$ line. One of these cannot be eliminated from the $\mathrm{CO}_{2}$ sample, since it is just the ${ }^{13} \mathrm{C}^{16} \mathrm{O}_{2}$ isotopologue, but its interference can be suppressed by cooling the gas, as already discussed. The other 2 interfering lines come from $\mathrm{N}_{2} \mathrm{O}$ and $\mathrm{O}_{3}$. Their upper concentration 


\section{Optical Detection of Radiocarbon Dioxide}

levels, which are acceptable to not affect the ${ }^{14} \mathrm{C}$ measurement, are, respectively, about $0.3 \mathrm{ppb}$ and $10 \mathrm{ppm}$. The removal from the $\mathrm{CO}_{2}$ sample of $\mathrm{N}_{2} \mathrm{O}$ below such a low concentration level may be not trivial: this issue has not been fully investigated. Instead, no problems are expected for reducing the $\mathrm{O}_{3}$ concentration below $10 \mathrm{ppm}$.

The most interesting comparison parameter among the different techniques is the achievable precision on a modern sample and the factor that limits this precision. LSC can achieve a precision as high as $0.5 \mathrm{pMC}$, but it is overcome by AMS, which can achieve even $0.3 \mathrm{pMC}$, and both of these techniques are limited by Poisson statistics of their counts. SCAR is not as precise as the other 2 techniques, as its current precision is only $2 \mathrm{pMC}$, and is limited by the achievable detection sensitivity. This can be expressed as the minimum absorption coefficient per unitary detection bandwidth $\alpha_{\min }\left[\mathrm{cm}^{-1} \mathrm{~Hz}^{-1 / 2}\right]$, which is inversely proportional to the optical $\mathrm{S} / \mathrm{N}$ ratio and to the cavity finesse.

Finally, in terms of footprint and cost, the LSC setup is the smallest and the least expensive; the SCAR setup is almost as small, and it is cheaper than the largest AMS machines. However, it is worth noting that AMS facilities involve apparatuses of different sizes and performances (aimed at different applications, not only ${ }^{14} \mathrm{C}$ ), whose footprint and cost approximately scale with the energy of accelerated ions (Kutschera 2005).

\section{Future Developments}

Future developments of our setup are summarized in Table 3. The main changes will involve the cell design, laser sources, and operating temperature. The material of the gas cell body will be changed from stainless steel to quartz, to have a smaller coefficient of thermal expansion (i.e. more stable length of the optical resonator) and walls more immune to contamination. The volume will be reduced from $\sim 8 \mathrm{~L}$ down to $0.7 \mathrm{~L}$ and hence, proportionally, the carbon mass from $70 \mathrm{mg}$ down to $6 \mathrm{mg}$. We have chosen not to reduce the 1-m cell length, to not lose absorption sensitivity, which is proportional to it, but the cell diameter only, from $100 \mathrm{~mm}$ down to $30 \mathrm{~mm}$. Such a value could be further reduced down to $\sim 10 \mathrm{~mm}$, but at the expense of a much more critical alignment of the mirrors. Indeed, in that case, since the beam waist on the mirror surface will be about $1.4 \mathrm{~mm}$, the light spots should be precisely centered on both mirrors, in order to avoid diffraction losses of the light inside the resonator. Since we will mount in the cell a couple of mirrors with slightly higher reflectivity at $4.5 \mu \mathrm{m}$ than the current ones, we expect to improve the optical finesse from about 11,000 to 15,000 and hence, proportionally, the absorption sensitivity.

Table 3 Future setup development.

\begin{tabular}{lll}
\hline & Present setup & Future setup \\
\hline Cell material & Stainless steel & Quartz \\
Volume $(\mathrm{L})$ & $\sim 8$ & $\sim 0.7$ \\
$\mathrm{C}$ mass $(\mathrm{mg})$ & $\sim 70$ & $\sim 6$ \\
Optical finesse & $\sim 11,000$ & $\sim 15,000$ \\
Laser source & Intracavity DFG & Tunable CW DFB-QCL1 \\
Absolute frequency reference & OFCS referenced to & ${ }^{14} \mathrm{C}^{16} \mathrm{O}_{2}$-locked \\
& quartz/Rb/GPS clock & CW DFB-QCL2 \\
Temperature (K) & 195 & 170 \\
$\frac{1}{\mathrm{~d} S}\left(\mathrm{~K}^{-1}\right)$ & $\sim 7 \times 10^{-4}$ & $\sim 2 \times 10^{-3}$ \\
$\overline{\mathrm{d} T}$ & & \\
Cooling method & Dry ice & Closed-cycle Stirling cryocooler \\
Footprint $\left(\mathrm{m}^{2}\right)$ & $\sim 2$ & $\sim 1$ \\
Cost $($ in thousands $€)$ & $\sim 300$ & $<100$ \\
\hline
\end{tabular}




\section{Galli et al.}

The current intracavity DFG source is very complex and requires an optical frequency-comb synthesizer (OFCS) referenced to a quartz/Rb/GPS clock to achieve the narrow linewidth needed for coupling light to the high-finesse cavity. ${ }^{6}$ We plan to do the same job with simpler laser sources, namely a couple of room-temperature $\mathrm{CW}$ distributed-feedback quantum cascade lasers (DFBQCLs) (Faist et al. 1994, 1997). We have recently demonstrated that these semiconductor laser sources have intrinsic linewidths as low as few hundred Hz (Bartalini et al. 2010, 2011). We have also succeeded in narrowing the free-running linewidth of a QCL from few hundred $\mathrm{kHz}$ down to a sub-kHz value (Cappelli et al. 2012), which is well below the width of the cavity mode. The first QCL will be frequency locked (and narrowed) to the ${ }^{14} \mathrm{C}^{16} \mathrm{O}_{2}$ target line, while the second one will be frequency scanned with respect to it when recording the absorption spectrum for the ${ }^{14} \mathrm{C}$ concentration measurement.

The operating temperature will be reduced from $195 \mathrm{~K}$ down to $170 \mathrm{~K}$ and the cooling method will change from dry ice to a closed-cycle Stirling cryocooler. This lower temperature will further reduce, by about a factor 30 , the interference from the $\left(05^{5} 1-05^{5} 0\right) \mathrm{P}(19)$ e line of the ${ }^{13} \mathrm{C}^{16} \mathrm{O}_{2}$ isotopologue, due to the steep temperature dependence of the Boltzmann population of the lower state involved in the transition. The only drawback will be a more critical dependence on temperature, about a factor 3 higher, for the linestrength of the target line. Moreover, the future cooling method with the Stirling cryocooler will be more flexible than the present one, which relies on dry-ice coolant at its fixed sublimation temperature. On a positive note, both the estimated footprint and cost of the future setup will be smaller than the present one, since the QCL sources are not only much simpler than the OFCS-locked intracavity DFG, but much cheaper, too.

Finally, if we inspect Equation 9, we can also discuss the overall uncertainty budget coming from the 3 terms multiplying the integral representing the measured spectral area. About the first term, absolute pressure can be measured by capacitance gauges at the $0.1 \%$ level. We can reasonably expect that, with the new cell design, temperature will be made uniform and stabilized within $0.5 \mathrm{~K}$, which means $\sim 0.3 \%$ uncertainty for the second term. Regarding the third term, by inspection of the 8th line in Table 3, we can estimate that thermal stability will yield a linestrength stability better than $0.1 \%$. We thus conclude that the overall precision in concentration measurements will be only limited by the current $\alpha_{\min }$. In the event of a significant improvement ( 1 order of magnitude or better), the ultimate achievable precision will be, in principle, as low as $0.3 \%$, i.e. the same as AMS. In that case, isotopic fractionation will be an issue to be considered, too. In order to correct the measured ${ }^{14} \mathrm{C}^{16} \mathrm{O}_{2}$ concentration for that effect, the concentration of the ${ }^{13} \mathrm{C}^{16} \mathrm{O}_{2}$ isotopologues will also have to be measured. This can be easily accomplished by using a conventional diode laser source emitting either at $2.8,2.1$, or $1.6 \mu \mathrm{m}$, where the ro-vibrational combination bands $v_{3}+v_{1}, v_{3}+2 v_{1}$ and $v_{3}+3 v_{1}$, respectively, lie. Even though the linestrengths of these bands are, respectively, about 1.5, 3 , and 5 orders of magnitude weaker than the fundamental $v_{3}$ band employed for the ${ }^{14} \mathrm{C}^{16} \mathrm{O}_{2}$ detection, the much higher natural abundance of ${ }^{13} \mathrm{C}$ with respect to ${ }^{14} \mathrm{C}$ (10 orders of magnitude), make this measurement easily accessible to the direct-absorption technique. A laser beam non-resonantly coupled to the SCAR cell can interact with the $\mathrm{CO}_{2}$ sample gas and the ${ }^{13} \mathrm{C}^{16} \mathrm{O}_{2}$ concentration can be retrieved from the measured absorption spectrum by using Equation 9 .

\section{CONCLUSION}

Our results pave the way for a new all-optical dating technique, moving from detection of highenergy $(\sim \mathrm{MeV})$ accelerated ions to the relatively easier detection of low-energy $(\sim 0.25 \mathrm{eV})$ absorbed

${ }^{6}$ With a free-spectral range of $150 \mathrm{MHz}$ and a finesse of $\sim 15,000$, the resonant mode width will be $\sim 10 \mathrm{kHz}$. 


\section{Optical Detection of Radiocarbon Dioxide}

photons. More generally, such a technique can be used to detect extremely rare molecules with a myriad of applications in many important fields.

\section{ACKNOWLEDGMENTS}

This work was financially supported by Regione Toscana through the projects CTOTUS, SIMPAS (for the position of I Galli within INO - CNR), and TEMART (for the position of M E Fedi within INFN) in the framework of POR-CReO/FESR 2007-2013, by Ente Cassa di Risparmio di Firenze, by CNR through the project Extreme Light Infrastructure, Italy (ELI-Italy) and by Laserlab-Europe consortium through the Joint Research Activity 2009-2012 JRA1:ALADIN.

\section{REFERENCES}

Bartalini S, Borri S, Cancio P, Castrillo A, Galli I, Giusfredi G, Mazzotti D, Gianfrani L, De Natale P. 2010. Observing the intrinsic linewidth of a quantum-cascade laser: beyond the Schawlow-Townes limit. Physical Review Letters 104:083904.

Bartalini S, Borri S, Galli I, Giusfredi G, Mazzotti D, Edamura T, Akikusa N, Yamanishi M, De Natale P. 2011. Measuring frequency noise and intrinsic linewidth of a room-temperature DFB quantum cascade laser. Optics Express 19(19):17,996-18,003.

Bennett CL, Beukens RP, Clover MR, Gove HE, Liebert RB, Litherland AE, Purser KH, Sondheim WE. 1977. Radiocarbon dating using electrostatic accelerators: negative ions provide the key. Science 198(4316): 508-10.

Cappelli F, Galli I, Borri S, Giusfredi G, Cancio P, Mazzotti D, Montori A, Akikusa N, Yamanishi M, Bartalini S, De Natale P. 2012. Subkilohertz linewidth room-temperature mid-IR quantum cascade laser using a molecular sub-Doppler reference. Optics Letters 37(23):4811-3.

Faist J, Capasso F, Sivco DL, Sirtori C, Hutchinson AL, Cho AY. 1994. Quantum cascade laser. Science 264(5158):553-6.

Faist J, Gmachl C, Capasso F, Sirtori C, Sivco DL, Baillargeon JN, Cho AY. 1997. Distributed feedback quantum cascade lasers. Applied Physics Letters 70(20): 2670.

Fedi ME, Cartocci A, Manetti M, Taccetti F, Mandò PA. 2007. The ${ }^{14} \mathrm{C}$ AMS facility at LABEC, Florence. $\mathrm{Nu}$ clear Instruments and Methods in Physics Research B 259(1):18-22.

Fedi ME, Bernardoni V, Caforio L, Calzolai G, Carraresi L, Manetti M, Taccetti F, Mandò PA. 2013. Status of sample combustion and graphitization lines at INFNLABEC. Radiocarbon, these proceedings.

Galli I, Bartalini S, Borri S, Cancio P, Giusfredi G, Mazzotti D, De Natale P. 2010. Ti:sapphire laser intracavity difference-frequency generation of $30 \mathrm{~mW} \mathrm{cw} \mathrm{ra-}$ diation around $4.5 \mu \mathrm{m}$. Optics Letters 35(21):3616-8.

Galli I, Bartalini S, Borri S, Cancio P, Mazzotti D, De Natale P, Giusfredi G. 2011. Molecular gas sensing below parts per trillion: radiocarbon-dioxide optical detection. Physical Review Letters 107:270802.
Giusfredi G, Bartalini S, Borri S, Cancio P, Galli I, Mazzotti D, De Natale P. 2010. Saturated-absorption cavity ring-down spectroscopy. Physical Review Letters 104:110801.

Kutschera W. 2005. Progress in isotope analysis at ultratrace level by AMS. International Journal of Mass Spectrometry 242(2-3):145-60.

Labrie D, Reid J. 1981. Radiocarbon dating by infrared laser spectroscopy. A feasibility study. Applied Physics 24(4):381-6.

Murnick DE, Peer BJ. 1994. Laser-based analysis of carbon isotope ratios. Science 263(5149):945-7.

Murnick DE, Dogru O, Ilkmen E. 2008. Intracavity optogalvanic spectroscopy. An analytical technique for ${ }^{14} \mathrm{C}$ analysis with subattomole sensitivity. Analytical Chemistry 80(13):4820-4.

Murnick DE, Dogru O, Ilkmen E. 2010. ${ }^{14} \mathrm{C}$ analysis via intracavity optogalvanic spectroscopy. Nuclear Instruments and Methods in Physics Research B 268(7-8): $708-11$.

Romanini D, Kachanov AA, Sadeghi N, Stoeckel F. 1997. CW cavity ring down spectroscopy. Chemical Physics Letters 264(3-4):316-22.

Rothman LS, Gordon IE, Barbe A, Benner DC, Bernath PF, Birk M, Boudon V, Brown LR, Campargue A, Champion J-P, Chance K, Coudert LH, Dana V, Devi VM, Fally S, Flaud J-M, Gamache RR, Goldman A, Jacquemart D, Kleiner I, Lacome N, Lafferty WJ, Mandin J-Y, Massie ST, Mikhailenko SN, Miller CE, Moazzen-Ahmadi N, Naumenko OV, Nikitin AV, Orphal J, Perevalov VI, Perrin A, Predoi-Cross A, Rinsland CP, Rotger M, Šimečková M, Smith MAH, et al. 2009. The HITRAN 2008 molecular spectroscopic database. Journal of Quantitative Spectroscopy and Radiative Transfer 110(9-10):533-72.

Schulze-König T, Dueker SR, Giacomo J, Suter M, Vogel JS, Synal H-A. 2010. BioMICADAS: compact next generation AMS system for pharmaceutical science. Nuclear Instruments and Methods in Physics Research B 268(7-8):891-4.

Synal H-A, Wacker L. 2010. AMS measurement technique after 30 years: possibilities and limitations of low energy systems. Nuclear Instruments and Methods in Physics Research B 268(7-8):701-7. 